\title{
Modelling non-paradoxical loss of information in black hole evaporation
}

\author{
Sujoy K. Modak and Daniel Sudarsky
}

\begin{abstract}
We give general overview of a novel approach, recently developed by us, to address the issue black hole information paradox. This alternative viewpoint is based on theories involving modifications of standard quantum theory, known as "spontaneous dynamical state reduction" or "wave-function collapse models" which were historically developed to overcome the notorious foundational problems of quantum mechanics known as the "measurement problem". We show that these proposals, when appropriately adapted and refined for this context, provide a self-consistent picture where loss of information in the evaporation of black holes is no longer paradoxical.
\end{abstract}

\section{Introduction}

The black hole information problem [15] is one of the most debated and controversial problems of theoretical physics, and has been the focus of considerable attention from various theoretical viewpoints during the last four decades (see [18] for a pedagogic introduction). We in fact note Paddy's recent proposal [16], [17] connected to this issue. The main reason behind this activity is the fact that while according to the unitary evolution law of Quantum Mechanics (QM), all information about a quantum state at any time is encoded in the state at any other time, the process

Sujoy K. Modak

KEK Theory Center, High Energy Accelerator Research Organization (KEK),

Tsukuba, Ibaraki 305-0801, Japan.

\&

Facultad de Ciencias - CUICBAS, Universidad de Colima, C.P. 28045, Colima, México

e-mail: sujoy@post.kek.jp

Daniel Sudarsky

Instituto de Ciencias Nucleares, Universidad Nacional Autónoma de México, México D.F. 04510,

México e-mail: sudarsky@nucleares.unam.mx 


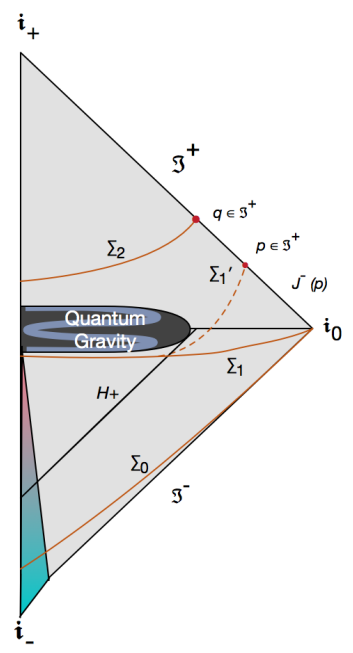

Fig. 1 Penrose diagram showing black hole formation and evaporation. The initial spacetime is Minkowskian at $\mathscr{I}^{-}$and at the end of Hawking evaporation Quantum Gravity (QG) resolves the would be classical singularity making the final spacetime asymptotically flat at $\mathscr{I}^{+}$.

of thermal black hole evaporation via Hawing radiation poses a "threat" to such an expectation, leading to the so called "paradox".

More specifically, let us consider the formation (by gravitational collapse) of a black hole and its subsequent evaporation (by Hawking effect) as shown in Fig. 1 . Let the initial state of the matter, defined on an initial Cauchy slice $\Sigma_{0}$, be characterized at the quantum level, by some pure, perhaps a coherent state. Under appropriate circumstances this matter collapses forming the black hole, and any quantum field, in this space-time will contribute to the Hawking radiation at late times. The radiation is characterized, in full, simply by the temperature and in particular it will be the same for any initial mass regardless the details of the initial quantum state. Thus unless some dramatic departure from the above picture takes place there would be no way to retro-dict the initial state from the former. The mapping from initial to final states would not be invertible and in particular it would fail to be represented in terms of a unitary operator.

The point is that, attempts to explain how the full state of the quantum fields unitarily related to the initial state would be encoded on late time hypersurfces such as $\Sigma_{2} \cup\left(J^{-}(q) \cap \mathscr{I}^{+}\right)$, have been unsuccessful until this date.

This, in turn, leads the majority of the physicists to believe that the fate of information and problem of unitarity in black hole evaporation is a unique situation where the unitarity of quantum evolution is in question . The recent finding of "firewall problem" [1] when considering a solution to the problem based on an approach known as based on "black hole complementarity" [32] is often presented as reflecting the tension between the unitarity of $\mathrm{QM}$ and equivalence principle of general relativity (GR). 
We, however, need to be a bit more careful because the mapping implied by quantum theory is expected to be unitary only if it refers to states defined on complete Cauchy hypersurfaces. The point is that, while the initial state was characterized on $\Sigma_{0}$, which is a true Cauchy hypersurface, the late time hypersurfaces would fail to be Cauchy hypersurfaces due to the presence of a space-time singularity deep within the black hole. This singularity (or more precisely a surface arbitrarily close to it) can in fact be considered as a boundary of the space-time and thus as a necessary component of any true late time Cauchy hypersurface. However the singularity is generally expected to be a feature of the fact that we have not incorporated the aspects of quantum gravity which should cure this singularity removing the need to include the extra space-time boundary. It is then and only then that we face a truly problematic situation [22].

Therefore the real tension between quantum theory and general relativity in the context of black hole formation and evaporation arises only when we view that quantum gravity will remove the singularity and thus the need to include a spacetime boundary. It is then and only then that we face something that could be considered truly paradoxical. We could now ask ourselves, what would be the problem of adopting the position that, all processes involving black hole evaporation do in fact break the unitarity of quantum evolution? As we see it, the problem with that position would be that, as we just saw, we would be working in a context where we imagine having incorporated aspects of quantum gravity in the discussion. Having done that, it seems inevitable to view processes such as black hole formation and evaporation as part of a larger class of processes, after all, the black hole concept is essentially a global one. That is the notion of Black Hole is not one that could be considered as lying at the basic formulation of the theory, which is expected to be described in terms of some fundamentally local degrees of freedom, rather that the global notions such as event horizons, or trapped surfaces that should appear only as secondary and emerging entities. In fact we should expect that black hole creation and evaporation should appear in the theory occurring also as virtual process contributing to essentially all physical processes, thus raising the question of when precisely can we expect to have an exact unitary evolution law as dictated by standard quantum theory.

In fact the black hole information issue, motivated the analysis in [2] where it was argued that loss of unitary would be need to accompanied by unacceptably large violations of energy conservation or of causality. A subsequent study of the issue reveled however that those arguments were not very robust, and that such expectations could be radically modified [35].

These considerations open the door to considering the question of information loss in the context of possible of modified versions of quantum theory involving departure from unitary evolution at the fundamental level.

In fact it is fair to say that all the approaches that have been proposed so far for the recovery of information (and the full quantum state that is unitarily related to the initial one) have not been successful as they end up adding to more problematic aspects to the picture. A big motivation of this rather "one way traffic" is the adherence to the notion that failure of unitarity in black hole evaporation would 
completely invalidate QM. However, as we advocate here, the situation is not as simple, because the violation of unitarity is not only not unexpected in quantum context but rather a common occurrence in any situation, normally thought as involving the collapse of wave-function due to whatever reason (natural interaction or laboratory measurement). That is, we have further motivation to consider the issue at hand, in connection with the so called general measurement problem in quantum theory.

Therefore, in contrast with the established tradition we will discuss here an approach based on the exact opposite possibility, i.e. the necessity of loss of information during black hole evaporation just as in most ordinary situations involving the quantum regime, thus "dissolving" the paradox.

This "dissolution" comes of course at the cost of losing quantum mechanical unitarity and one might worry whether we would lose with it all the successes of standard QM. We furthermore note that from the foundational point of view regarding quantum theory, there have been various proposal of a modified version of quantum dynamics incorporating a spontaneous collapse of the wave-function to elevate QM from a theory of measurement to the theory of reality (see [27] for the terminology) in a manner that the subjective role of an observer becomes removed and one can treat QM objectively without introducing any extraneous notion of observer as an essential entity shaping reality. This collapse process is spontaneous and stochastic, and it is implemented in such a manner that the well established and experimentally successful predictions of quantum mechanics remain unaffected, while a gradual difference in the predictions appears as the quantum system's size approaches that of macroscopic object (for an account of ongoing experimental endeavor, see [4] ). Thus, in building such type of theories, the aim is to resolve the measurement problem and eliminate various in-built two level descriptions of reality in Copenhagen interpretation; such as, micro/macro, classical/quantum, system/apparatus, system/observer, system/environment etc.

In this article, we will not discuss in detail any of these proposals (for that we refer the reader to the papers [10]-[13], [23]-[27], [5, 28, 29, 33, 34] as well as review articles [3, 4]), but we shall use some specific models and provide a concrete example offering a overview of the manner in which such models can deal with the information problem in black holes leading to a picture where the associated breakdown of unitarity is a part and parcel of the (modified) general quantum mechanical evolution.

\section{Measurement problem and models of wave-function collapse}

According to the Copenhagen interpretation of quantum mechanics there are two distinct evolution rules for the quantum state/wave-function of a system. First, a continuous evolution as dictated by the Schrödinger equation and valid while the system is left alone and free from observations, and the second, a discontinuous and stochastic jump to one of the eigenstates (dictated by Born probability rule) of some self-adjoint operator in the Hilbert space once measurement by an external observer 
takes place. As characterized by R. Penrose, the first case is a unitary evolution or the $U$-process, while the second case is a reduction or the $R$-process, and measurement is a notion that separates these two processes. The problem is that within the standard view of QM, measurement does not have any kind of rigorous definition, nor is it clear when exactly it is performed during a evolution. In fact such vague and artificial division has been sharply criticized by J. Bell [7]

...If the theory is to apply to anything but highly idealized laboratory operations, are we not obliged to admit that more or less 'measurement-like' processes are going on more or less all the time, more or less everywhere? Do we not have jumping then all the time?

Also, it is an in-built aspect of the standard presentations, that without observer or some entity which is "measuring" the system, no specific outcome is presupposed in QM.

Within the community, working on the foundation of quantum mechanics, there are of course diverse viewpoints regarding the measurement problem. These include the Many World Interpretations, in its various forms, the Bohmian Mechanics program representing a reliance on nonlocal hidden variables, and the proposals for unifying the $U$ and $R$ processes, referred as the Dynamical Reduction Program (DRP), pioneered by Pearle, Ghirardi, Rimini and Weber.

The DRP first included a discrete process of collapse in the wave-function/quantum state, driven by an additional non-unitary and stochastic term modifying the Schrödinger evolution.

The basic idea in those proposals is that the evolution of systems with very small number of degrees of freedom is dominated by the standard part of the dynamics resulting in very small deviations from that predicted by standard theory, ensuring the reproduction of the stupendous success of quantum theory in high precision laboratory experiments whereas, the non-standard terms becomes dominant when a rather large number of degrees of freedom appear in a state representing a rather delocalized quantum superposition, thus ensuring the rapid collapse to one or the other of the classical looking components of Schrödinger cat states.

This feature ensures that when, what is normally called a measurement is performed, the system is driven to one or the other eigenstates of the apparatus' pointer's position simply because such pointer consists of a macroscopically large number of degrees of freedom. That is, as a result of the new general dynamical law the theory reproduces the standard predictions of quantum theory regarding the measurement of the appropriate self-adjoint operator. The first and is simplest successful model of this kind known as Ghirardi-Rimini-Weber (GRW) theory [11], which was later improved to deal with identical particles in a scheme that makes wavefunction collapse a continuous process and known as the CSL theory [12, 26, 27]. Their recent advances in this direction have resulted in proposals for relativistic version of both type of theories [5, 28, 29, 33]. In this article we restrict ourselves to the non-relativistic framework of CSL theory to adress the issue of black hole information and for the relativitic framework we refer the interested reader to our recent work [6]. 


\subsection{CSL theory: non-relativistic setting}

The non-relativistic version of the CSL theory [12, 26, 27] is, at this point, much better explored than the relativistic counterpart and it is defined by following two equations: (i) A stochastically modified Schrödinger equation, whose solution is:

$$
|\psi, t\rangle_{w}=\hat{\mathscr{T}} e^{-\int_{0}^{t} d t^{\prime}\left[i \hat{H}+\frac{1}{4 \lambda_{0}}\left[w\left(t^{\prime}\right)-2 \lambda_{0} \hat{A}\right]^{2}\right]}|\psi, 0\rangle
$$

where $\hat{\mathscr{T}}$ is the time-ordering operator, $w(t)$ is a random, white noise type classical function of time and its probability distribution is given by the second equation, (ii) the Probability Distribution (PD) rule:

$$
P D w(t) \equiv_{w}\langle\psi, t \mid \psi, t\rangle_{w} \prod_{t_{i}=0}^{t} \frac{d w\left(t_{i}\right)}{\sqrt{2 \pi \lambda_{0} / d t}} .
$$

Thus the standard Schrödinger evolution and corresponding changes in the state corresponding to a "measurement" of the operator $\hat{A}$ are unified and the dynamics does not allow any superluminal signaling. In the non-relativistic limit, for a single particle, the proposal assumes that there is a spontaneous and continuous reduction characterized by $\hat{A}=\hat{\mathbf{X}}_{\delta}$, where $\hat{\mathbf{X}}_{\delta}$ is a suitably smeared position operator (with the smearing characterized by the scale $\delta$ ). This smearing of the position operator is required to avoid an uncontrolled increase in energy associated with a point-like collapse event. The resultant theory can be applied to all situations without invoking any measurement device or observer. This framework can be easily extended to multiparticle system by choosing a set of operators representing each particle so that everything, including, the apparatuses are treated quantum mechanically. The final theory, thus seems to successfully address the measurement problem and completely overlook various two level descriptions in Copenhagen interpretation.

Since the final outcome of the collapse of an individual state vector is uncertain, it is useful to consider a collection of identical initial state and describe the evolution of an ensemble in the language of a density matrix. The CSL evolution of density matrix can be derived from a Lindblad type equation with a solution [12, 26, 27]

$$
\rho(t)=\hat{\mathscr{T}} e^{-\int_{0}^{t} d t^{\prime}\left[i(\stackrel{\hat{H}}{\rightarrow}-\hat{H}]+\frac{\lambda}{2}[\stackrel{\hat{A}}{\rightarrow}-\stackrel{\hat{A}}{\leftarrow}]^{2}\right]} \rho(0)
$$

where the arrows mean the operators act on the left or right of $\rho(0)$.

\section{Callan-Giddings-Harvey-Strominger (CGHS) Model}

We choose the 2 dimensional version of black hole formation and evaporation, provided by the CGHS model [8], to exhibit an explicit realization of our proposal. The CGHS action is given by 


$$
S=\frac{1}{2 \pi} \int d^{2} x \sqrt{-g}\left[e^{-2 \phi}\left[R+4(\nabla \phi)^{2}+4 \Lambda^{2}\right]-\frac{1}{2}(\nabla f)^{2}\right]
$$

where $\phi$ is the dilaton field, $\Lambda^{2}$ is a constant, and $f$ is a real scalar field, representing matter. The Penrose diagram of CGHS model is shown in Fig. 2. Before the

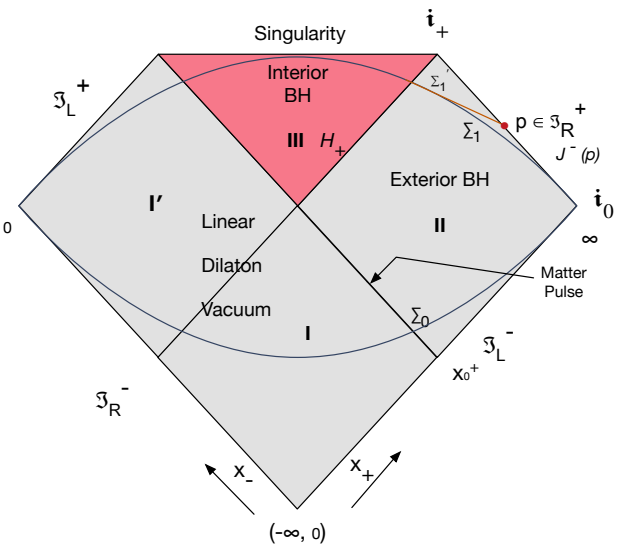

$\mathfrak{i}$

Fig. 2 Penrose diagram for CGHS spacetime. Minkowskian and black hole regions are separated by a sharp gravitational collapse of null matter like photon.

gravitational collapse $\left(x^{+}<x_{0}^{+}\right)$, the metric is Minkowskian, usually known as the dilaton vacuum (region I and I'), given by $d s^{2}=-\frac{d x^{+} d x^{-}}{-\Lambda^{2} x^{+} x^{-}}$, whereas, at $x^{+}>x_{0}^{+}$ it is represented by the black hole metric (region II, III) ${ }^{1} d s^{2}=-\frac{d x^{+} d x^{-}}{\frac{M}{\Lambda}-\Lambda^{2} x^{+}\left(x^{-}+\Delta\right)}$. In regions I and I', natural Minkowskian coordinates are $y^{+}=\frac{1}{\Lambda} \ln \left(\Lambda x^{+}\right), y^{-}=$ $\frac{1}{\Lambda} \ln \left(-\frac{x^{-}}{\Delta}\right)$, with $-\infty<y^{-}<\infty ;-\infty<y^{+}<\frac{1}{\Lambda} \ln \left(\Lambda x_{0}^{+}\right)$. On the other hand, on the $\mathrm{BH}$ exterior (region II), where physical observers might exist, one has the coordinates $\sigma^{+}=\frac{1}{\Lambda} \ln \left(\Lambda x^{+}\right)=y^{+}, \sigma^{-}=-\frac{1}{\Lambda} \ln \left(-\Lambda\left(x^{-}+\Delta\right)\right)$ and the metric is $d s^{2}=-\frac{d \sigma^{+} d \sigma^{-}}{1+(M / \Lambda) e^{\Lambda\left(\sigma^{-}-\sigma^{+}\right)}}$with $-\infty<\sigma^{-}<\infty$ and $\sigma^{+}>\sigma_{0}^{+}=\frac{1}{\Lambda} \ln \left(\Lambda x_{0}^{+}\right)$. It is easy to check the asymptotic flatness of the black hole metric by introducing Schwarzschild like time $t$ and space $r$ coordinates [19, 20] using $\tanh (\Lambda t)=T / X$ and $-\frac{1}{\Lambda^{2}}\left(e^{2 \Lambda r}-M / \Lambda\right)=T^{2}-X^{2}$.

The quantum description of the field $f$ can be made using two different natural bases. In the asymptotic past $\left(\mathscr{I}_{L}^{-} \cup \mathscr{I}_{R}^{-}\right.$or in) region, the basis mode functions are chosen to be: $u_{\omega}^{R}=\frac{1}{\sqrt{2 \omega}} e^{-i \omega y^{-}}$and $u_{\omega}^{L}=\frac{1}{\sqrt{2 \omega}} e^{-i \omega y^{+}}$, with $\omega>0(R$ and $L$ indicate

${ }^{1}$ more precisely, region I', although flat, is also part of the interior of the event horizon as nothing in that region can ever reach $\mathscr{I}_{R}^{+}$ 
right and left moving modes respectively). The tensor product of respective vacuum state defines the in vacuum $\left(\left|0_{i n}\right\rangle_{R} \otimes\left|0_{i n}\right\rangle_{L}\right)$. In the asymptotic future (out region) we use a basis of modes that have support in the outside (exterior) and inside (interior) to the event horizon. The mode functions in the exterior to the horizon are: $v_{\omega}^{R}=\frac{1}{\sqrt{2 \omega}} e^{-i \omega \sigma^{-}} \Theta\left(-\left(x^{-}+\Delta\right)\right)$ and $v_{\omega}^{L}=\frac{1}{\sqrt{2 \omega}} e^{-i \omega \sigma^{+}} \Theta\left(x^{+}-x_{0}^{+}\right)$. Similarly, and in order to have a complete out basis, one chooses a set of modes for the black hole interior. Usually for the left moving modes, one maintains the same functional form as before, and for the right moving modes one takes: $\hat{v}_{\tilde{\omega}}^{R}=\frac{1}{\sqrt{2 \tilde{\omega}}} e^{i \tilde{\omega} \sigma_{i n}^{-}} \Theta\left(x^{-}+\Delta\right)$.

It is convenient to replace the above delocalized plane wave modes by a complete orthonormal set of discrete and sharply localized wave packets modes [9, 14],

$$
v_{n j}^{L / R}=\frac{1}{\sqrt{\varepsilon}} \int_{j \varepsilon}^{(j+1) \varepsilon} d \omega e^{2 \pi i \omega n / \varepsilon} v_{\omega}^{L / R},
$$

where the integers $j \geq 0$ and $-\infty<n<\infty$. These wave packets are peaked about $\sigma^{+/-}=2 \pi n / \varepsilon$ with width $2 \pi / \varepsilon$ respectively.

The non-trivial Bogolyubov transformations are only relevant in the right moving sector, and are the ones that in fact account for the Hawking radiation. The initial state, corresponding to the vacuum for the right moving modes, and the left moving pulse (which leads to the formation of the black hole) $\left|\Psi_{i n}\right\rangle=\left|0_{i n}\right\rangle_{R} \otimes \mid$ Pulse $\rangle_{L}$ can be expanded in the out basis:

$$
N \sum_{F_{n j}} C_{F_{n j}}\left|F_{n j}\right\rangle^{e x t} \otimes\left|F_{n j}\right\rangle^{i n t} \otimes|P u l s e\rangle_{L}
$$

where the states $\left|F_{n j}\right\rangle$ are characterized by the finite occupation numbers $\left\{F_{n j}\right\}$ for each corresponding mode $n, j ; N$ is a normalization constant, and the coefficients $C_{F_{n j}}$ 's are determined by the Bogolyubov transformations.

\section{Gravitational induced collapse of wave-function and loss of information}

As we have mentioned in the very beginning, the wave-function collapse model, as given by the CSL theory, needs to be adapted in order to be applicable to the problem at hand. One novel addition to the already developed CSL theory, is our hypothesis that gravitational field enhances the rate of wave-function collapse. This can be achieved by making the collapse rate as a function of the Weyl curvature scalar $W_{a b c d} W^{a b c d}$ as first suggested by Okon and Sudarsky [21]. That is, even in the absence of any measuring device/observer, in a spacetime region with enormously large curvature (such as inside the horizon and towards the center of a black hole), quantum superpositions are increasingly broken in a stochastic manner (provided by the CSL stochasticity) and produces similar effects as those caused by an external measurement usually considered in a laboratory context. It should be mentioned that 
such an effect of gravitation on quantum mechanics was strongly advocated by R. Penrose in several of his works (see, for instance, [30, 31]) and we consider those as a guiding path leading to our explicit demonstration.

We first note that in the multi-particle system, the CSL evolution (1) is generalized to

$$
|\psi, t\rangle_{w_{\alpha}}=\hat{\mathscr{T}} e^{-\int_{0}^{t} d t^{\prime}\left[i \hat{H}+\frac{1}{4 \lambda_{0}} \sum_{\alpha}\left[w_{\alpha}\left(t^{\prime}\right)-2 \lambda_{0} \hat{A}_{\alpha}\right]^{2}\right]}|\psi, 0\rangle,
$$

where $\alpha$ is an index labeling the set of particles. Our aim is to consider a CSL evolution (analogous to $(6)$ applied to a field theory with the index $\alpha$ having a different meaning) of the initial state (5). Moreover we will treat CSL as an interaction term, so that the free Hamiltonian will be set to zero, i.e $H=0$ in (6). As we are using this equation in the context of QFT in curved spacetime, we need to choose a new operator, that must be constructed using the field operator and its derivatives. One such operator is the number operator (for right moving modes) defined in the interior Fock basis times the identity for the exterior Fock basis:

$$
\hat{A}^{\alpha}=\hat{N}_{n j}^{i n t} \otimes \mathbb{I}^{e x t}
$$

for all $n, j$, where $\hat{N}_{n j}^{i n t}=\hat{N}_{n j}^{\text {int }(R)} \otimes \mathbb{I}^{\text {int }(L)}$ and $\mathbb{I}^{\text {ext }}=\mathbb{I}^{\text {ext }(L)} \otimes \mathbb{I}^{\text {ext }(R)}$. This ensures that the collapse will make the wave-packet to peak about particular values of $n$ and $j$ (which will be picked randomly depending on the specific realization of the noise $w_{n j}(t)$ ). In the standard CSL type evolution (6) it takes, strictly speaking, an infinite amount of time to fully collapse the wave-function to an eigenstate of the collapse operator due to the finite value of the collapse parameter $\lambda_{0}$. It is to be noted that the experimental bounds on $\lambda_{0}$ come from laboratory based experiments that are, of course, performed in a spacetime regions where curvature is negligible. Here we make a hypothesis that the collapse rate is in fact sensitive to the local curvature, so that a more general expression must have the following form

$$
\lambda\left(W^{2}\right)=\lambda_{0}\left(1+\left(W^{2} / \mu^{2}\right)^{\gamma}\right)
$$

where $\mu$ is an appropriate scale and $\gamma \geq 1$. One anticipated effect of this, is to generate an complete effective collapse of the quantum state, taking place in a finite time interval.

In the particular case of 2D models the Weyl scalar vanishes identically so instead, for this special case, we assume that $\lambda$ is determined by the Ricci scalar. Thus, for this specific case (8) is replaced by,

$$
\lambda(R)=\lambda_{0}\left(1+(R / \mu)^{\gamma}\right)
$$

where, for the CGHS black hole $R=\frac{4 M \Lambda}{M / \Lambda-\Lambda^{2}\left(T^{2}-X^{2}\right)}$. The Kruskal time and space coordinates are respectively $T=\frac{x^{+}+x^{-}+\Delta}{2}$ and $X=\frac{x^{+}-x^{-}-\Delta}{2}$. Next we provide a brief account of calculation, further details can be found in [19], [20].

Foliation of the spacetime: To implement CSL in the black hole model we need to foliate the spacetime with an appropriately defined spacelike/Cauchy slices, as 


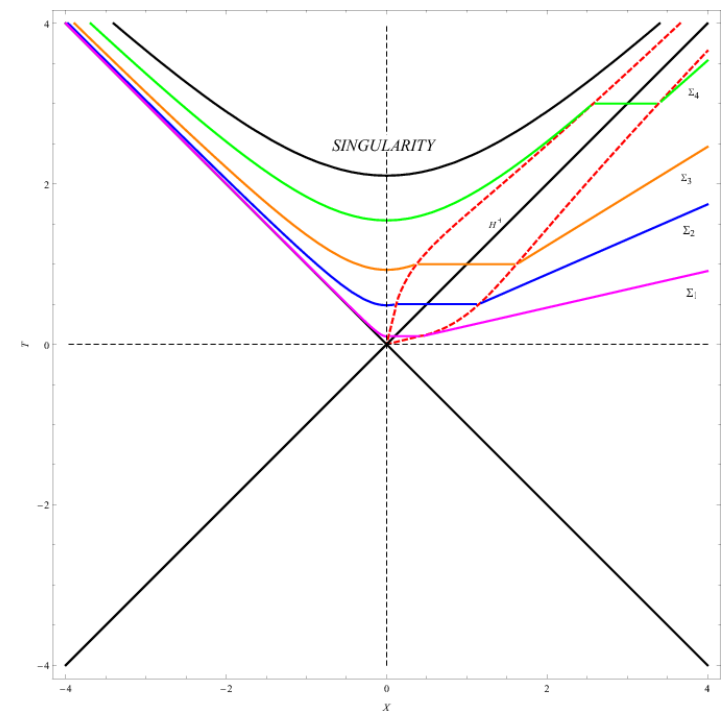

Fig. 3 Foliation of the CGHS spacetime by Cauchy slices. All the plots have fixed $M / \Lambda^{3}=4.42$, while $T=0.1,0.5,1,3$ (in magenta, blue, orange and green) as the joining $T=$ const. curves.

plotted in Fig. 3, and use the time evolution from one slide to the other. As there is no natural notion of "time" in the relevant regions of these spacetimes, we take a convenient time parameter $(\tau)$ that will be used to characterize the quantum state evolution, according to the CSL dynamics.

We define the Cauchy slices to be Schwarzschild $r=$ const. inside the horizon and Schwarzschild $t=$ const . outside the horizon, and join them by surfaces with Kruskal $T=$ const. . The intersection curves joining the family of Cauchy slices $r=$ const . and $T=$ const . at one end (inside horizon) is chosen to be $T_{1}(X)=$ $\left(X^{2}+\frac{M}{\Lambda^{3}} e^{-2 \Lambda / \sqrt{X}}\right)^{1 / 2}$, whereas, at the other end (outside horizon) the intersection curve $T_{2}(X)$ can be found just by using a reflection about the event horizon $T=X$. We fix the value of the "time" parameter $\tau$ as the value of the coordinate $T$ on the intersection of $r=$ const. with $X=0$ (or $T$ axis), so that the Ricci scalar is expressed as $R=\frac{4 M \Lambda}{M / \Lambda-\Lambda^{2} \tau^{2}}$. It is now clear that $R$ diverges for some finite value of $\tau=\tau_{s}=\frac{M^{1 / 2}}{\Lambda^{3 / 2}}$ corresponding to the divergence of $R$ that characterizes the singularity.

Evolution of the quantum state: In standard CSL theory state is evolved according to the equation (6), which, in the present situation is subjected to the changing collapse parameter 9 , that become a function of time parameter $\tau$ and, the collapse operators (7). The initial state for the right moving modes, traveling from $\mathscr{I}_{R}^{-}$to $\mathscr{I}_{R}^{+}$is denoted by the "in" vacuum for right moving modes, which can be expressed in the "out" basis according to (5) (we leave for the moment the "pulse" which is left moving and forms the black hole). The evolution equation of the state vector for right moving modes become 


$$
|\Psi, \tau\rangle_{R}=N \sum_{F} C_{F_{n j}} e^{-\int_{o}^{\tau} d \tau^{\prime}\left[\frac{1}{4 \lambda} \sum_{n, j}\left(w_{n j}-2 \lambda F_{n j}\right)^{2}\right]}\left|F_{n j}\right\rangle_{R}^{i n t} \otimes\left|F_{n j}\right\rangle_{R}^{e x t}
$$

where $F_{n j}$ is the eigenvalue of the operator $\hat{N}_{n j}^{i n t}$ while acting on the state $|F\rangle^{i n t}$. As $\tau$ approaches $\tau_{s}$ the Cauchy slices tend to reach the spacetime singularity, and $R$ diverges. This divergence in $R$ makes the integral in (10) divergent, and thus the initial state collapses to a state with definite quantum numbers $n, j$, giving

$$
\lim _{\tau \rightarrow \tau_{s}}|\Psi, \tau\rangle_{R}=N C_{F_{n_{0} j_{0}}}\left|F_{n_{0} j_{0}}\right\rangle_{R}^{i n t} \otimes\left|F_{n_{0} j_{0}}\right\rangle_{R}^{e x t},
$$

on the hypersurface $\Sigma_{1}$ (Fig. 4 as it approaches the singularity. As the level of each mode's excitation depends on the realization of the stochastic value $w_{n j}\left(\tau_{s}\right)$, the final state after collapse, although remains pure, it is undetermined.

Evolution at the ensemble level as given by the density matrix: To account for the lack of predictability of the final state we consider a large collection of systems all prepared in the same initial state and use an ensemble description in terms of a density matrix. The evolution equation becomes

$$
\rho_{R}(\tau)=N^{2} \sum_{F, G} e^{-\frac{\pi}{\Lambda}\left(E_{F}+E_{G}\right)} e^{-\sum_{n j}\left(F_{n j}-G_{n j}\right)^{2} \int_{\tau_{0}}^{\tau} d \tau^{\prime} \frac{\lambda\left(\tau^{\prime}\right)}{2}}|F\rangle_{R}^{i n t} \otimes|F\rangle_{R}^{e x t}\left\langleG | _ { R } ^ { i n t } \otimes \left\langle\left. G\right|_{R} ^{e x t} .\right.\right.
$$

Therefore, near the singularity (on $\Sigma_{1}$ in Fig. 44, as $\lambda$ diverges in the exponential factor, the result is a diagonal density matrix of the form (omitting $n, j$ from subscript for simplified notation and putting explicit expression for $C_{F}$ ):

$$
\lim _{\tau \rightarrow \tau_{s}} \rho_{R}(\tau)=N^{2} \sum_{F} e^{-\frac{2 \pi}{\Lambda} E_{F}}|F\rangle_{R}^{i n t} \otimes|F\rangle_{R}^{e x t}\left\langleF | _ { R } ^ { i n t } \otimes \left\langle\left. F\right|_{R} ^{e x t},\right.\right.
$$

where $E_{F}=\sum_{n j} \omega_{n j} F_{n j}$ is the total energy of the final excited state.

The description of the state vector and density matrix is complete once we include the left moving matter pulse, so that

$$
\begin{aligned}
& \left.\lim _{\tau \rightarrow \tau_{s}}|\Psi, \tau\rangle_{R}=N e^{-\frac{\pi}{\Lambda} E_{F_{0}}}\left|F_{0}\right\rangle_{R}^{\text {int }} \otimes\left|F_{0}\right\rangle_{R}^{\text {ext }} \otimes \mid \text { Pulse }\right\rangle_{L} \\
& \lim _{\tau \rightarrow \tau_{s}} \rho(\tau)=N^{2} \sum_{F} e^{-\frac{2 \pi}{\Lambda} E_{F}}|F\rangle_{R}^{\text {int }} \otimes|F\rangle_{R}^{\text {ext }}\left\langle\left. F\right|_{R} ^{\text {int }} \otimes\left\langle\left. F\right|_{R} ^{\text {ext }} \otimes\right| \text { Pulse }\right\rangle_{L}\left\langle\text { Pulse }\left.\right|_{L}(15)\right.
\end{aligned}
$$

where $F_{0}$ is understood as a specific particle excited state $F_{n_{0} j_{0}}$ and $E_{F_{0}}$ is the energy of this state.

Quantum gravity $(Q G)$ and resolution of singularity: To pass from the hypersurface $\Sigma_{1}$ to $\Sigma_{f}$, in Fig. 4. one has to rely on a theory of QG which is likely to involve giving up the classical notion of "spacetime". In the absence of any fully workable theory of that kind, we make a few natural assumptions about QG theory, namely that - (i) it resolves the singularity and leads on the other side, to a regime describable with standard classical notions of space-time, (ii) it does not lead to arbitrarily large violations of standard conservation laws such a energy conservation. If so, then QG makes following operation after combining the negative energy 


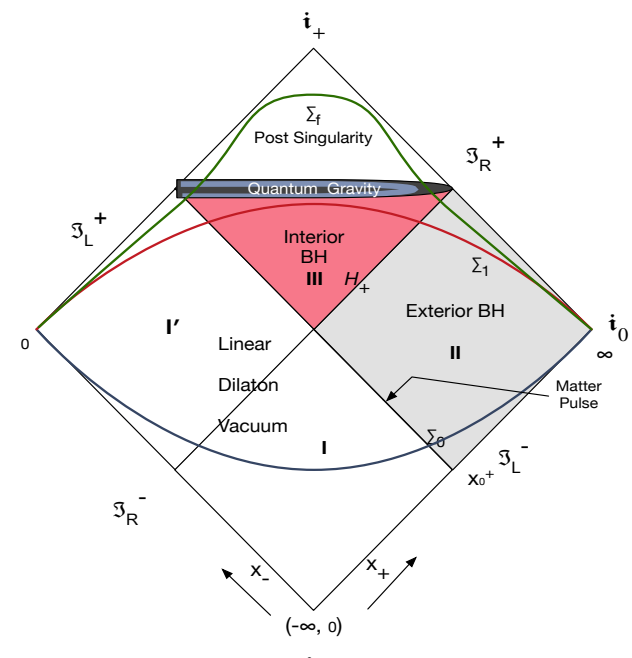

i

Fig. 4 Penrose diagram of CGHS spacetime with Quantum Gravity (QG) region.

state $\left|F_{0}\right\rangle_{R}^{i n t}$ (which is complementary to Hawking radiation) with the positive matter $\mid$ Pulse $\rangle$

$$
\left|F_{0}\right\rangle_{R}^{i n t} \otimes|P u l s e\rangle_{L} \rightarrow|p . s\rangle
$$

where $|p . s\rangle$ is a post-singularity quantum state with almost vanishing energy and residing as the complement of Hawking radiation near $\mathscr{I}_{R}^{+}$on $\Sigma_{f}$. Then on the final hyper-surface $\Sigma_{f}$, the quantum state and the density matrix becomes

$$
\begin{aligned}
& |\Psi\rangle_{R}=N e^{-\frac{\pi}{\Lambda} E_{F_{0}}} \otimes\left|F_{0}\right\rangle_{R}^{\text {ext }} \otimes|p . s\rangle \\
& \rho=N^{2} \sum_{F} e^{-\frac{2 \pi}{\mu} E_{F}}|F\rangle_{R}^{\text {ext }}\left\langle\left. F\right|_{R} ^{\text {ext }} \otimes \mid p . s\right\rangle\langle p . s| . \\
& \quad=\rho_{\text {thermal }}^{\text {ext }} \otimes \mathbb{I}_{\text {p.s. }}^{\text {ext }}
\end{aligned}
$$

The resulting picture, therefore, indicates that the final state on the Cauchy slice $\Sigma_{f}$, for an individual system is pure, yet undetermined while at the ensemble level it is proper mixed state as the density matrix is clearly thermal on the asymptotic regime times a state with a very low energy ( and idealized to be vacuum ) characterizing the remaining portion of $\Sigma_{f}$, (which is then taken to be a portion of flat spacetime). Thus the complete evolution is non-unitary and information is lost, mainly in the interior of the black hole, as a consequence of wave-function collapse. There is of course nothing paradoxical in this picture. 


\section{Discussions}

We have put forward a novel proposal involving gravitational influenced wavefunction collapse which we showed can account for the enormous loss of information in black hole evaporation which thus leads to a dissolution of the so called "paradox". On a broader perspective, this opens up a rather interesting possibility: that in the energy scale interpolating between, say, the current LHC (or the Standard Model) energy scale (about $10 \mathrm{TeV}$ ) and somewhere below the QG scale $\mathrm{e}^{2}\left(10^{16}\right.$ $\mathrm{TeV}$ ), there could be important effects describable in the context of the standard model of particle physics adapted to the modified quantum field theory constructed on curved space-times with the additional feature of gravitational induced quantum state reduction, as provided, say, by one of the relativistic collapse proposals [5, 28, 29, 33, 34]. Could it be, for instance, that the issue of the radiative corrections induced quantum instability of the Higgs potential are modified by the introduction of such modifications? Could we do with a scheme where supper-symmetry is not needed and have similar benefits arising, instead, from the effects of quantum collapse? We believe this line of inquire might offer interesting insights and modify the perspectives for physics beyond standard model, and perhaps the expectations for phenomenology of quantum gravity.

Acknowledgements We want to thank our collaborators E. Okon, L. Ortíz, I. Peña, D. Bedingham for their involvement and contribution towards this project. SKM is an International Research Fellow of Japan Society for the Promotion of Science. This research is partly funded by Grant-inAid to JSPS fellows (KAKENHI-PROJECT-15F15021). DS acknowledges partial financial support from DGAPA-UNAM project IG100316 and by CONACyT project 101712.

\section{References}

1. Almheiri, A., Marolf, D., Polchinski, J., Sully, J.: Black Holes: Complementarity or Firewalls? JHEP 1302, 062 (2013).

2. Banks, T., Susskind, L., Peskin, M.E.: Difficulties for the evolution of pure states into mixed states. Nucl. Phys. B 244, 125 (1984).

3. Bassi, A., Ghirardi, G. C.: Dynamical reduction models. Phys. Rept. 379, 257 (2003).

4. Bassi, A., Lochan, K., Satin, S., Singh, T.P., and Ulbricht, H.: Models of Wave-function Collapse, Underlying Theories, and Experimental Tests, Rev. Mod. Phys. 85, 471 (2013).

5. Bedingham, D.: Relativistic state reduction model. J. Phys. Conf. Ser. 306, 012034 (2011).

6. Bedingham, D., Modak, S. K., Sudarsky, D.: Relativistic collapse dynamics and black hole information loss. Phys.Rev. D 94, 045009, (2016) [arXiv:1604.06537 [gr-qc]].

7. Bell, J.: Against Measurement, (1990).

8. Callan, C.G., Giddings, S.B., Harvey J. A., Strominger, A.: Evanescent black holes. Phys. Rev. D 45, R1005 (1992).

9. Fabbri, A., Navarro-Salas, J.: Modeling Black Hole Evaporation. Imperial College Press, London, 2005.

${ }^{2}$ up to the regime where we expect the validity of standard space-time notions as provided by General Relativity. 
10. Ghirardi, G., Rimini, A., Weber, T.: A model for a unified quantum description of macroscopic and microscopic systems. In A. L. Accardi (ed.) Quantum Probability and Applications, p. p. 223-232, Springer, Heidelberg (1985).

11. Ghirardi, G., Rimini, A., Weber, T.: Unified dynamics for microscopic and macroscopic systems. Phys.Rev. D 34, 470 (1986).

12. Ghirardi, G., Pearle, P., Rimini, A.: Markov-processes in Hilbert-space and continuous spontaneouslocalization of systems of identical particles. Phys. Rev. A 42, 7889 (1990).

13. Ghirardi, G., Grassi, R., Pearle, P.: Relativistic dynamical reduction models: General framework and examples. Found. Phys. (J.S. Bell's 60th birthday issue) 20, 1271 (1990).

14. Giddings, S. B., Nelson, W. M.: Quantum emission from two-dimensional black holes. Phys. Rev. D 46, 2486 (1992).

15. Hawking, S.W.: Breakdown of Predictability in Gravitational Collapse. Phys. Rev. D 14, 2460 (1976).

16. Lochan, K., Chakraborty, S., Padmanabhan, T.: Information retrieval from black holes. arXiv: 1604.04987 [gr-qc].

17. Lochan, K., Padmanabhan, T.: Extracting information about the initial state from the black hole radiation. Phys. Rev. Lett. 116, no. 5, 051301 (2016).

18. Mathur, S. D.: The Information paradox: A Pedagogical introduction. Class. Quant. Grav. 26, 224001 (2009).

19. Modak, S.K., Ortíz, L., Peña, I., Sudarsky, D.: Non-Paradoxical Loss of Information in Black Hole Evaporation in a Quantum Collapse Model. Phys. Rev. D 91, no. 12, 124009 (2015).

20. Modak, S.K., Ortíz, L., Peña, I., Sudarsky, D.: Black hole evaporation: information loss but no paradox. Gen. Rel. Grav. 47, no. 10, 120 (2015)

21. Okon, E., Sudarsky, D.: Benefits of Objective Collapse Models for Cosmology and Quantum Gravity. Found. of Phys. 44 114-143, (2014).

22. Okon, E., and Sudarsky, D.: The Black Hole Information Paradox and the Collapse of the Wave Function. Found. Phys. 45, 461-470 (2015).

23. Pearle, P.: Reduction of the state vector by a nonlinear Schrodinger equation. Phys. Rev. D 13, 857 (1976).

24. Pearle, P.: Towards explaining why events occur. Int. J. Theor. Phys. 18, 489 (1979).

25. Pearle, P.: Combining stochastic dynamical state-vector reduction with spontaneous localization. Phys. Rev. A 39, 2277-2289 (1989).

26. Pearle, P.: Collapse models. [arXiv: quant-ph/9901077].

27. Pearle, P.: Collapse Miscellany. Contributed in 80th birthday Issue of Yakir Aharanov. [arXiv:1209.5082 [quant-ph]]

28. Pearle, P.: Toward a relativistic theory of statevector reduction. In Sixty-Two Years of Uncertainty, edited by A. Miller (Plenum, New York, 1990) p. 193-214.

29. Pearle, P.: A Relativistic Dynamical Collapse Model. [arXiv:1412.6723 [quant-ph]].

30. Penrose, R.: (chapter) Newton, quantum theory and reality. In Three Hundred Years of Gravitation, Cambridge University Press (1989) (p. 17).

31. Penrose, R.: On Gravity's role in Quantum State Reduction. Gen. Relativ. and Gravit., 28, 581 (1996).

32. Susskind, L., Thorlacius, L., Uglum, J.: The Stretched horizon and black hole complementarity. Phys. Rev. D 48, 3743 (1993)

33. Tumulka, R.: A relativistic version of the Ghirardi-Rimini-Weber model. J. Stat. Phys. 125, 821 (2006).

34. Tumulka, R.: On spontaneous wave function collapse and quantum field theory. Proc. R. Soc. A 462, 1897 (2006).

35. Unruh, W.G., and Wald, R. M.: On evolution laws taking pure states to mixed states in quantum field theory. Phys.Rev. D 52, 2176-2182 (1995). 